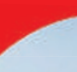

\section{ON THE RECORD \\ CThe pain was immediate and felt like my body was on fire.)}

Endurance swimmer Lewis Pugh (pictured) after his 19-minute swim $\stackrel{ }{~}$ at the North Pole, when he braved water temperatures of $-1.8^{\circ} \mathrm{C}$ to raise awareness of melting polar ice.

\section{SCORECARD}

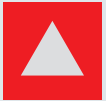

Low-sugar water melons Diabetics and dieters will be pleased to learn that US geneticists have bred water melons with $50 \%$ less sugar than normal.

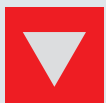

\section{High-fat friends} A 30-year study monitoring some 12,000 Americans concludes that they were $57 \%$ more likely to become obese if one of their close friends became obese too, suggesting that social ties help to spread the obesity epidemic.

\section{ZOO NEWS}

\section{Prolific panda}

Huamei, a US-born giant panda now living at a reserve in Sichuan, China, has delivered her third set of twins, boosting China's captive giant-panda population by nearly $1 \%$.

\section{NUMBER CRUNCH}

74 watts is the power needed to display a full-screen version of the Google homepage on a conventional cathode-ray monitor.

\section{3,000 megawatt-}

hours is the energy that would be saved worldwide each year if everyone switched to blackle.com, a new website that allows users to do Google-powered searches from a dark page that uses only 59 watts.

\section{$25 \%$ is the proportion of} computers worldwide that still use cathode-ray monitors. This figure is declining all the time, prompting cynics to argue that 'dark searching' is merely a gimmick.

Sources: The Times, USDA, N. Engl. J. Med., blackle.com; ecolron, Reuters

\title{
Carbon sinks threatened by increasing ozone
}

Rising levels of ozone pollution over the coming century will erode the ability of plants to absorb carbon dioxide from the atmosphere, a new climate-modelling study predicts.

Ozone is already known to be a minor greenhouse gas, but the new calculations highlight another, indirect way in which it is likely to influence global warming by 2100 . High levels can poison plants and reduce their ability to photosynthesize, says Stephen Sitch of the UK Met Office's Hadley Centre for Climate Prediction and Research in Exeter.

Whereas ozone high in the stratosphere shields Earth from solar ultraviolet rays, high levels of ozone closer in are toxic to plants

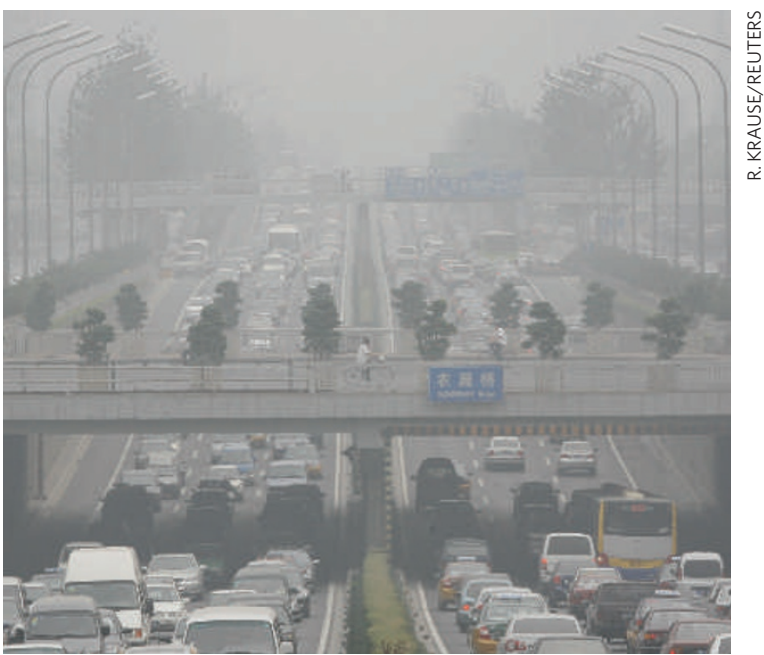

Beijing smog is a sign of rising ozone pollution. and animals. Ozone is generated when oxides of nitrogen - chiefly from vehicle exhausts and fossil-fuel power stations - react with other chemicals in the air.

Many of the world's most polluted areas routinely endure ozone concentrations higher than 40 parts per billion, enough to damage plant life. By the end of the century, virtually all the world's populated areas are predicted to be above this threshold.

Plant growth is a vital carbon sink, estimated to suck up around a quarter of the $\mathrm{CO}_{2}$ emitted into the atmosphere. "This study combines knowledge about ecosystem function with atmospheric chemistry, and that's never been done before," says Sitch. "No one has considered the detrimental effect of ozone on plants."

The issue is complicated by the intricate interplay between rising $\mathrm{CO}_{2}$ levels, which boost plant growth, and increasing ozone, which stunts it. Some ecosystem models had predicted that the rise in $\mathrm{CO}_{2}$ expected over the coming century might be offset by the expected

\section{Diamonds 'melted' inside an onion}

Diamonds may not be for ever: researchers think they have seen, for the first time, the molten form of what is arguably the world's hardest material.

Jianyu Huang of Sandia

National Laboratories in New Mexico heated diamond, at high pressure, to more than $2,000^{\circ} \mathrm{C}$ inside microscopic hollow shells of carbon and watched the diamond soften (J. Y. Huang Nano Lett. doi:10.1021/nl0709975; 2007).

Huang describes this as quasi-melting. It is not true melting, he says, because the diamond particles don't become liquid carbon, but instead flicker between crystal forms by continually melting and instantly refreezing in a different conformation.

This is the closest anyone has ever come to directly melting diamond. No one really knows what molten carbon looks like. One form, graphite, seems to break down into liquid-like blobs when heated to high temperatures, although they have not been examined while still molten. But until now, molten diamond had not been achieved.

A key problem is that when diamond is heated to very high temperatures, at atmospheric pressure, it turns into graphite rather than melting. To overcome this, Huang's team used structures known as 'carbon onions' to create very high pressures and temperatures.

These roughly spherical concentric shells of graphite- 
boost in the growth of forests and swamps. But factoring in ozone means that it looks less likely that this carbon sink will grow fast enough to keep pace with the increasing emissions.

Eva Pell, who studies the effects of ozone on plants at Pennsylvania State University in University Park and who was not involved in the study, finds the results credible. "There is no doubt that ozone reduces $\mathrm{CO}_{2}$ fixation," she says. "It makes sense that the adverse effects of ozone would be dampened by elevated $\mathrm{CO}_{2}$, and the ability of plants to serve as carbon stores in an elevated $\mathrm{CO}_{2}$ environment would be reduced by elevated ozone."

In 1901, according to the researchers' calculations, plant growth was responsible for storing 113 billion tonnes of carbon worldwide. By 2100 , this figure is predicted to be 171 billion tonnes - without ozone it would be more than 200 billion tonnes, they say. Their results are published online this week (S. Sitch et al. Nature doi:10.1038/nature06059; 2007).

Different plant species vary in their sensitivity to ozone, and these figures reflect predictions based on a fairly high average level of toxicity, says team member Bill Collins of the Met Office. But even in the researchers' low-sensitivity calculations, there's likely to be a dent of around 15 billion tonnes in overall carbon sequestration as a result of the effects of ozone.

"The bottom line is that ozone is a greenhouse gas, so it's known to contribute to the greenhouse effect. Our study says you should double that predicted contribution," says Collins.

Unlike most greenhouse gases, ozone is a short-lived, regional pollutant that can be tackled at an individual level using catalytic converters, for example, to reduce precursors to ozone, Sitch suggests.

Michael Hopkin

\section{Memory seen in the making}

The physical changes that occur when the brain makes a new memory have been observed for the first time, say researchers, who hope to go on to map the distribution of memory across brain regions.

Gary Lynch of the University of California, Irvine, and his colleagues examined the junctions between neurons - synapses - in three dimensions using a technique called restorative deconvolution microscopy (RDM). This consists of a sensitive light microscope with computer algorithms that analyse light scattered above and below the focal point, producing a three-dimensional 'trace' of an object's structure.

In previous work, the group developed a fluorescent marker that attaches to synapses in the brain that have recently undergone a certain type of neuronto-neuron connection believed to be responsible for encoding memory, called long-term potentiation (LTP) (L. Y. Chen et al. J. Neurosci. 27, 5363-5372; 2007).

The team exposed live rats to a novel environment and allowed them to learn its layout. They then removed the animals' brains to examine the hippocampus - a region involved in memory - using RDM to observe individual synapses. A second group of rats was shown the new environment but not allowed to explore it before their brains were examined.
Only rats that had undergone learning and memory acquisition showed new synaptic growth, Lynch says (V. Fedulov et al. J. Neurosci. 27, 8031-8039; 2007). And the hippocampal synapses to which the LTP fluorescent marker attached were $50 \%$ larger than other synapses not involved in LTP.

Furthermore, when the group looked at hippocampal slices from a third group of rats, which had been allowed to learn the same new environment but been given a drug to block LTP, the synapses showed no new growth, and were similar to those of the second group. This indicates that the new synaptic growth observed in the study is a result of LTP, Lynch asserts.

"We saw that the synapses had actually changed shape as a result of the new memory," Lynch says. "They went from oval to circles, which have a greater surface area." He now aims to use the technique to see which other areas of the brain might be involved in memory.

Being able to look at memory at the synaptic level is a major advance, says Mark Bear, a neuroscientist at the Massachusetts Institute of Technology in Cambridge. But he hesitates to accept the conclusions Lynch's team has drawn. "I don't think it's been proven that these [synaptic] changes represent the memory," he says.

Kerri Smith

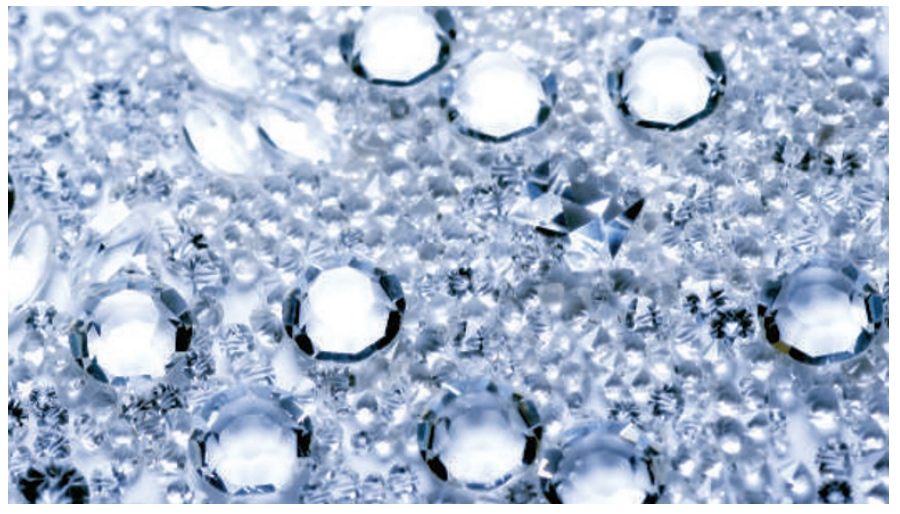

Quick change: molten diamond flickers between crystal forms.

like carbon act as high-pressure cells that shrink when blasted with a beam of electrons. As they contract, the material in their centres is squeezed to very high pressures. Previous studies by Florian Banhart of the Institute for Physical Chemistry in Mainz, Germany, and Pulickel Ajayan of the Rensselaer Polytechnic Institute, New York, showed that graphite-like carbon in the onion's centre can be converted to diamond in these chambers (F. Banhart and P. M. Ajayan Nature 382, 433-435; 1996).

Huang's team used carbon onions with a carbon nanotube attached to the outside. They wired up the nanotube and heated the tube-onion composite while irradiating it with electrons. Huang estimates that this created temperatures of more than $2,000^{\circ} \mathrm{C}$ and pressures of around 400,000 atmospheres at the core.
As the onions shrank, the carbon at the centre was transformed from graphite to diamond. When this got hot enough, it adopted the fluctuating quasi-molten state, Huang explains. Ultimately, he hopes, it will be possible to make fully molten diamond, so that the onions have liquid carbon cores.

But not everyone is confident that the diamond is really melting. Although Banhart says the new work is interesting, he thinks that the carbon may instead be switching between diamond and graphite - each time it returns to diamond, the crystals will look different, he says.

Philip Ball 